\title{
USO FORMATIVO DE INSTRUMENTOS DE EVALUACIÓN EN SECUNDARIA, LENGUA EXTRANJERA - INGLÉS: REDACCIONES
}

Formative use of assessment instruments in secondary education, foreign language English: writing

Uso formativo de ferramenta de avaliação em educação secundária, língua estrangeira inglês: redação

\section{Patricia Rivera Blanco (1)}

Jorge Garrote García (2)

(1) IES Gaspar Melchor de Jovellanos, Fuenlabrada. Teléfono: +34 916971565. Correo electrónico: patriciarivbla@gmail.com

(2) IES Lancia (León), España. Teléfono: +34 987259800. Correo electrónico: jorge.garrote@hotmail.com

\begin{abstract}
Resumen
Uno de los problemas que más conciernen a la comunidad educativa reside en la falta de claridad y concreción a la hora de evaluar las producciones de los alumnos; y de manera más específica las dificultades en la realización y evaluación de producciones escritas en lengua extranjera. A través de esta propuesta se busca demostrar que si se establecen unos criterios concretos y concisos que permitan tanto a alumnos como a profesores la evaluación de estas actividades de forma objetiva, es posible, no solo aportar algo de claridad y de justicia en los procesos de evaluación, sino también ayudar a los alumnos a ser más conscientes de la calidad de su trabajo y facilitar que puedan seguir mejorando. A través de un instrumento de evaluación entregado al inicio del proceso de enseñanza-aprendizaje (PEA) y de procesos de autoevaluación, coevaluación y heteroevaluación, tanto alumnos como docentes podrán tener claros los aspectos en los que centrarse así como los resultados que se esperan.

Palabras clave: redacción; lengua extranjera; evaluación formativa; instrumento de evaluación
\end{abstract}




\begin{abstract}
One of the main concerns for the educational community is the lack of clarity and precision when assessing the productions of the students; and more specifically the struggle when creating and assessing written tasks in the foreign language subject. Our proposal tries to prove that when the criteria are specific and concise both teachers and students are able to assess these tasks in an unbiased way. It is possible, not only to provide some clarity and fairness in the assessment processes, but also to help our students to become more aware of the quality of their work and to help them to keep improving. By means of an assessment instrument handed in at the beginning of the learning process and through different stages of self-assessment, peer assessment and teacher assessment, both students and teachers will be clear about the aspects to focus on as well as the expected results.
\end{abstract}

Keywords: Writing; foreign language; formative assessment; assessment instrument

\title{
Resumo
}

Um dos problemas que mais preocupam a comunidade educativa é a falta de clareza e precisão ao avaliar produções dos alunos; e mais especificamente dificuldades na condução e avaliação do trabalho escrito em uma língua estrangeira. Através desta proposta pretende mostrar que se houver critérios concretos e concisos que permitem que os alunos e professores avaliar essas atividades estabelecidas de forma objectiva, é possível fornecer alguns processos com clareza e justiça, mas também ajudar os alunos a se tornar mais consciente da qualidade do seu trabalho e facilitar-lhes continuar a melhorar. Através de um instrumento de avaliação previsto no início do ensinoaprendizagem (PEA) e processo de auto-avaliação, avaliação pelos pares e heteroavaliação, os alunos e professores terão áreas claras no qual o foco e os resultados esperados.

Palavras-chave: Redação; língua estrangeira; avaliação formativa; ferramenta de avaliação

\section{Introducción}

Una situación común para casi todos los docentes es recibir trabajos de nuestros alumnos que distan mucho de lo esperado. En estos momentos siempre surge la duda 
sobre cuánta responsabilidad es nuestra en relación a que el resultado no sea el adecuado. En la mayoría de estos casos, los alumnos no saben de dónde procede su calificación ni en qué fallaron, no son conscientes de porqué su trabajo ha obtenido una nota u otra y se sienten en muchas ocasiones frustrados por no saber qué deberían haber hecho para haber podido realizar un trabajo mejor. La legislación en vigor dedica un amplio apartado a la evaluación objetiva, sin embargo, son muchas las aulas en las que sigue mandando el "ojímetro" o evaluaciones subjetivas y poco claras que no convencen ni a alumnos ni a profesores. En este contexto de indeterminación es donde entran en juego los instrumentos de evaluación formativa entregados al inicio del PEA.

\section{Contextualización}

Esta experiencia se llevó a cabo en el IES Gaspar Melchor de Jovellanos (Fuenlabrada) con alumnos de $2^{\circ}$ de la ESO y $1^{\circ}$ de Bachillerato en la asignatura de Lengua Extranjera: Inglés. Tras observar diversas dificultades al realizar producciones escritas (writing) como por el ejemplo: el desconocimiento del modo de organizarlas, su estructuración o su adecuación al nivel de exigencia propio del curso; se decidió crear un instrumento que ayudara a los alumnos (ver tabla 1, modificada de Rivera y Garrote, 2014, 2014b). Dicho instrumento de evaluación permitió establecer una serie de elementos clave con varios subniveles perfectamente descritos e identificables que permitieran guiarles en la realización de las tareas escritas.

Ya que los alumnos no estaban acostumbrados a trabajar con este tipo de instrumentos, el primer paso fue entregárselo y analizarlo. Una vez todos los alumnos comprendieron los distintos aspectos a valorar, así como los distintos niveles establecidos dentro de cada apartado, éstos realizaron una composición escrita siguiendo los elementos de la plantilla. Dicha composición escrita seguramente ya era mejor que las que acostumbraban a hacer, ya que el alumno sabía perfectamente qué aspectos se iban a valorar. Quizás esta es la función más importante de este instrumento, que les permite mejorar y aprender más y con esa función precisamente fue creado, buscando una verdadera evaluación formativa.

Una vez finalizada la composición escrita, se realizó un proceso de autoevaluación (Arsyad, 2015) y coevaluación entre los alumnos utilizando la escala de valoración entregada al inicio. 
Finalmente, el profesor realizó una evaluación del documento que fue comparada con las realizadas por el propio alumno y sus compañeros.

Tabla 1

Rúbrica utilizada

\begin{tabular}{|c|c|c|c|c|}
\hline \multicolumn{2}{|l|}{ ASSSSMENT SCALE - WRITING } & \multirow[t]{2}{*}{1} & \multirow[t]{2}{*}{2} & \multirow[t]{2}{*}{... } \\
\hline GRAMMATICAL STRUCTURES & 25 & & & \\
\hline Includes at least 3 grammatical structures and tenses correctly used & 25 & & & \\
\hline Includes three different tenses and two grammatical structures correctly used & 20 & & & \\
\hline Includes two grammatical tenses and two grammatical structures correctly used & 15 & & & \\
\hline Includes two grammatical tenses but only one complex structure, correctly used & 10 & & & \\
\hline Includes only one grammatical structure or tense correctly used & 5 & & & \\
\hline All grammar contents are incorrectly used & Fail & & & \\
\hline CONTENTS & 15 & & & \\
\hline Deals with all the required aspects & 15 & & & \\
\hline Only deals with some of the aspects required & 10 & & & \\
\hline Deals only with one aspect in the whole writing & 5 & & & \\
\hline Includes different aspects than those required & 0 & & & \\
\hline STRUCTURE & 10 & & & \\
\hline Follows the given structure with linking words and has one paragraph for each main idea & 10 & & & \\
\hline Follows structure but paragraphs are mixed up/no clear paragraphing or use of linking words limited/poor & 5 & & & \\
\hline Does not follow the require structure or has not included any linking word & fail & & & \\
\hline VOCABULARY & 20 & & & \\
\hline Includes a variety of synonyms $(+5)$ related to topic without repetition of words (exc. Common verbs) & 20 & & & \\
\hline Includes some words (5) with some mistakes in the use or without broad vocabulary & 15 & & & \\
\hline There are some words (4) related to the topic but very basic, with some mistakes & 10 & & & \\
\hline The student used only 2-3 words related to the topic but incorrectly or too simple & 5 & & & \\
\hline CREATIVITY & 10 & & & \\
\hline Contents are original and imaginative or presented in an interesting way. Overall idea is interesting & 10 & & & \\
\hline The writing is interesting but not original/personal. Some aspects are copied from sample & 7 & & & \\
\hline The structure, contents and ideas are exactly the same as in the sample & 4 & & & \\
\hline The writing does not attract the reader's attention at all & 0 & & & \\
\hline SPELLING & 10 & & & \\
\hline There are no spelling mistakes (less than 3 ) & 10 & & & \\
\hline There are 3-5 spelling or punctuation mistakes & 5 & & & \\
\hline There are more than 5 spelling mistakes & 0 & & & \\
\hline OVERALL IMPRESSION & 5 & & & \\
\hline Neat and clear handwriting & 5 & & & \\
\hline \multicolumn{5}{|l|}{ TASK } \\
\hline Does not follow the instructions, does not hand in task or extension is $10 \%$ different & Fail & & & \\
\hline
\end{tabular}

\section{Diseño y desarrollo}

Para el diseño de un instrumento de evaluación que se adecuara a las necesidades de la tarea, del nivel y del grupo en primer lugar se analizaron distintas propuestas, como rúbricas, listas de control, escalas verbales o escalas de valoración. Finalmente tras un análisis del currículum del área, así como del contexto concreto se optó por la creación de una escala de valoración, ya que esta permitía establecer distintos aspectos a valorar de la producción escrita, otorgando a cada uno de ellos un valor diferente sin la necesidad de que el número de indicadores de cada aspecto a valorar fuera el mismo en todos los casos, como sí ocurre con las rúbricas (López-Pastor \& Pérez-Pueyo, 2017).

Se tuvieron en cuenta los distintos aspectos gramaticales, léxicos, estructurales y de contenido que se trabajan en la asignatura para después establecer una 
serie de subniveles adaptados a la etapa y curso concretos. Como dicho instrumento de evaluación también iba a ser utilizado como instrumento de calificación, se decidió asignar una puntuación numérica a cada aspecto y nivel alcanzado de manera que los propios alumnos pudieran saber perfectamente la nota que su trabajo merecía incluso antes de que nosotros la valorásemos.

\section{Evaluación y conclusiones}

Tras el uso continuado de este instrumento de evaluación y calificación se pudieron observar resultados positivos en la mayoría de los alumnos.

En primer lugar, contar con este instrumento permitió que los alumnos tuvieran claros los aspectos claves de la actividad así como los criterios que se iban a valorar tras su entrega del producto final, permitiéndoles en todo momento ajustar su trabajo al resultado que desearan obtener. Además, se detectó una mayor predisposición a la realización de este tipo de tareas, ya que los alumnos contaban con unas pautas claras sobre lo que se les estaba exigiendo. Por último, se consiguió evitar que los alumnos tuvieran cualquier tipo de reclamación, ya que las calificaciones seguían escrupulosamente lo establecido en la escala de valoración. Además, las valoraciones realizadas por ellos mismos, por sus compañeros y por el profesor eran muy similares e incluso iguales en la mayor parte de los casos.

Sin embargo, también se fueron detectando casos concretos que llevaron a plantearse un reajuste numérico de algunos de los aspectos. Se dieron dos casos de alumnos que, aún cumpliendo con la mayoría de los aspectos no obtenían calificaciones ajustadas a lo esperado dado que elementos como la originalidad y aspectos de contenido que habían incluido en sus producciones podían estar sujetos a subjetividades. También nos encontramos con aspectos como los gramaticales o léxicos que exigieron el incremento de subniveles para adecuar el nivel de exigencia curricular al de la tarea. Precisamente este proceso de diseño del instrumento, con la consiguiente fase de puesta en práctica e identificación de posibles errores, debe culminar con una modificación de los errores observados y una posterior reevaluación del instrumento para conseguir que sea lo más justo, objetivo y transparente posible y sobre todo, que sirva para que el alumno pueda seguir mejorando y aprendiendo. 


\section{Referencias}

Arsyad, A. (2015). Using self-assessment technique to improve students' writing skill of the eleventh grade. ELTS Journal, 3(2) 1-11.

López-Pastor, V.M., \& Pérez-Pueyo, A. (Coords.) (2017). Buenas prácticas docentes. Evaluación formativa y compartida en educación: experiencias en todas las etapas educativas. León: Servicio de publicaciones de la Universidad de León.

Rivera, P., \& Garrote, J. (2014). Assessment scale for written productions (ESO). Recuperado de https://drive.google.com/file/d/0B6zM2sfGO-

\section{LsS3UxbUpoQjdBRmM/view}

Rivera, P., \& Garrote, J., (2014). Assessment scale for written productions (BACHILLERATO).

Recuperado

de https://drive.google.com/file/d/0B6zM2sfGO-LsNINVaVZud0hjWVU/view 